\title{
Influence of Thermal Radiation on MHD Fluid Flow over a Sphere
}

\author{
M. Prameela ${ }^{1}$ (D), Dasari Venkata Lakshmi ${ }^{2}$ (D) , Jithender Reddy Gurejala ${ }^{3, *(D)}$ \\ 1 Department of Mathematics, P.V.P. Siddhartha Institute of Technology, Vijayawada,520007, Andhra Pradesh, India \\ 2 School of Computer Science and Engineering, VIT AP University, 522237, Andhra Pradesh, India \\ 3 Department of Mathematics, VNR Vignana Jyothi Institute of Engineering \& Technology, Bachupally, Hyderabad, \\ 500090, Telangana State, India \\ * Correspondence: jithendergurejala@gmail.com (G.J.R.);
}

Scopus Author ID 57189054458

Received: 14.09. 2021 ; Revised: 20.10.2021 ; Accepted: 24.10.2021; Published: 21.11.2021

\begin{abstract}
The current perusal investigation was carried out for the result of a chemical reaction and Schmidt number on magnetohydrodynamic fluid flow towards a Sphere with Rosseland approximation. The Roseland estimate is utilized to portray the radiative heat transition in the energy condition. The crucial equations of continuity, thermal and solutal boundary layers are reassembled into sets of nonlinear models. The highly nonlinear partial differential models are converted into a nonlinear ordinary differential structure through the proper dimensionless quantities. The numerical arrangements of standard differential structures have been procured by applying the fourth-order Runge-KuttaFehlberg strategy with shooting technique through MATHEMATICA software. The quantities of physical interest are graphically presented and discussed in detail. Correlation with past writing results is additionally done and is discovered to be excellent concurrence with those distributed before.
\end{abstract}

Keywords: MHD; chemical reaction; Sphere; Rosseland approximation.

(C) 2021 by the authors. This article is an open-access article distributed under the terms and conditions of the Creative Commons Attribution (CC BY) license (https://creativecommons.org/licenses/by/4.0/).

\section{List of Symbols}

$u, v:$ Velocity components in $x$ and $y$ axes respectively $(\mathrm{m} / \mathrm{s})$

$r: \quad$ The radial distance from the symmetrical axis to the surface of the sphere $(\mathrm{m} / \mathrm{s})$

$g: \quad$ Acceleration due to gravity $\left(\mathrm{m} / \mathrm{s}^{2}\right)$

$a: \quad$ Radius of the sphere $(m)$

$B_{o}: \quad$ Strength of Magnetic field (Tesla)

$q_{r}: \quad$ Radiative heat flux

$C_{p}: \quad$ The specific heat at constant pressure

$D$ : The coefficient of Mass diffusivity

$k^{*}: \quad$ Mean absorption coefficient

$G r$ : Thermal Grashof number (or) Grashof number for heat transfer

$K c$ : Dimensional Chemical reaction Parameter

$M: \quad$ Magnetic field parameter

$R: \quad$ Thermal radiation parameter

$C f$ : Skin-friction coefficient

Sc : Schmidt number

$x, y:$ Cartesian coordinates axis $(m)$

https://biointerfaceresearch.com/ 
$f: \quad$ Dimensionless stream function

$f^{\prime}: \quad$ Fluid velocity $(\mathrm{m} / \mathrm{s})$

Pr : Prandtl number

$T_{\infty}$ : Temperature of the fluid far away from the sphere $(K)$

$C_{w}:$ Dimensional concentration at the sphere $\left(\mathrm{mol} / \mathrm{m}^{3}\right)$

$\mathrm{Nu}: \quad$ Nusselt number coefficient

$S h:$ Sherwood number coefficient

$C_{\infty}: \quad$ Dimensional ambient volume fraction $\left(\mathrm{mol} / \mathrm{m}^{3}\right)$

$q_{w}:$ The surface (wall) heat flux

$m_{w}$ : The surface (wall) mass flux

$C: \quad$ Fluid concentration $\left(\mathrm{mol} / \mathrm{m}^{3}\right)$

$T: \quad$ Fluid temperature $(K)$

$T_{w}: \quad$ Temperature at the surface $(K)$

\section{Greek symbols}

$\kappa: \quad$ Thermal conductivity of base fluid

$\phi: \quad$ Nanoparticle concentration $\left(\mathrm{mol} / \mathrm{m}^{3}\right)$

$\eta$ : Dimensionless similarity variable

$\theta: \quad$ Dimensionless temperature $(K)$

$\alpha: \quad$ Thermal diffusivity $\left(\mathrm{m}^{2} / \mathrm{s}\right)$

$v: \quad$ Kinematic viscosity $\left(m^{2} / s\right)$

$\psi: \quad$ Stream function

$\tau_{w}: \quad$ Shear stress

$\gamma: \quad$ Chemical reaction parameter

$\beta_{T}$ : Volumetric coefficient of thermal expansion

$\beta_{C}$ : Volumetric coefficient of concentration expansion

$\sigma: \quad$ Electrical conductivity

$\rho$ : Density

$\sigma_{o}:$ Stefan-Boltzmann constant

\section{Superscript}

': $\quad$ Differentiation w.r.t. $\eta$

\section{Subscripts}

$f: \quad$ Fluid

$w: \quad$ Condition on the sphere

$\infty$ : Ambient Conditions 


\section{Introduction}

As of late, magnetohydrodynamics boundary layer stream and heat transfer of electrically conducting liquids have different science, designing, and mechanical applications such as petrol businesses, precious stone development, geothermal designing, atomic reactors, fluid metals, streamlined features, and metallurgical cycles. This relies upon whether they happen at an interface or as a solitary stage volume response. In all-around blended frameworks, the response is heterogeneous in the event that it happens at an interface and homogeneous in the event that it happens in an arrangement. By and large of substance responses, the response rate relies upon the centralization of the species itself. Ahmadi and Willing [1] studied the heat transfer measurement experimentally in -based water nanofluids and developed a computational fluid dynamics model using the Eulerian-Lagrangian approach to study the nature of both the laminar and turbulent flow fields of the fluid and the dispersed nanoparticles. The third-grade nanofluidic flow features towards a Riga surface via the Cattaneo-Christov theory are described by Naseem et al. [2]. Ismail et al. [3] analyzed dissipative impacts in a stagnant-point flow along a shrinking surface. Soomro et al. [4] examined the stagnation-point flow involving nanofluid through a moving sheet with nonlinear radiation and heat generation/absorption. Ghalambaz et al. [5] studied local thermal nonequilibrium analysis of conjugate free convection within a porous enclosure occupied with Ag$\mathrm{MgO}$ hybrid nanofluid. Veera Krishna and Chamkha [6] investigated the diffusion-thermo, Hall, radiation-absorption, and ion slip effects on magnotohydrodynamic natural convective flow of nano-fluids past a semi-infinite permeable moving plate with the presence of a constant heat source. Thameem Basha et al. [7] reported the characteristics of nonlinear radiation and induced magnetic field on the forced convective Falkner-Skan flow of SWCNH/EG and water nanofluid over a wedge, plate, and stagnation point. Kumar et al. [8] inspected the steady flow of electrical conducting, viscous, incompressible, and optically thin fluid flow over a vertical plate with magnetohydrodynamic mixed convection, viscous dissipation, and thermal radiation. Veera Krishna and Chamkha [9] explored the Hall and ion slip influence on the magnetohydrodynamic convective flow of elastico-viscous fluid through the porous medium between two rigidly rotating parallel plates with time fluctuating sinusoidal pressure gradient. Veera Krishna et al. [10] explored the Hall and ion slip influence on the unsteady magnetohydrodynamic free convective rotating flow over an exponential accelerated inclined plate entrenched in a saturated porous medium with the effect of angle of inclination, variable temperature, and concentration. Ramesh et al. [11] scrutinized heat transport through a stagnation-point hybrid nano liquid flow past a permeable cylinder. Menni et al. [12] explored the thermal and hydrodynamic analysis of forced-convective flows of pure ethylene glycol, pure water, and water-ethylene glycol mixture, as base fluids spread by $\mathrm{Al}_{2} \mathrm{O}_{3}$ nano-sized solid particles, over a constant temperature surfaced rectangular cross-section channel with the influence of various effects. Ganesh Kumar et al. [13] studied Reiner-Philippoff fluid flow over a heated surface through the theory of Cattaneo-Christov for heat diffusion. Wakif et al. [14] investigated thermo-magneto-hydrodynamic irreversibilities arising in the dissipative flows of weakly conducting fluids past over a moving Riga plate. Bhowmik et al. [15] studied transient natural convection heat transfer analyses from a horizontal cylinder. Jena et al. [16] studied the chemical reaction effect on MHD viscoelastic fluid flow over a vertical stretching sheet with a heat source/sink. Anil Kumar et al. [17] studied the effects of soret, dufour, hall current, and rotation on MHD natural convective heat and mass transfer flow past an accelerated vertical 
plate through a porous medium. Shankar Goud et al. [18] analyzed the thermal radiation and Joule heating effects on a magnetohydrodynamic Casson nanofluid flow in the presence of chemical reaction through a nonlinear inclined porous stretching sheet. Heat and mass transfer on unsteady MHD Oscillatory flow of blood through porous arteriole has been explained by Veera Krishna et al. [19]. Seth et al. [20] discussed the numerical simulation of the Newtonian heating effect on unsteady MHD flow of Casson fluid past a flat vertical plate considering the impact of viscous dissipation, Joule heating, thermal diffusion, and nth-order chemical reaction. Recently researchers [21-31] studied MHD mixed convective flow on a sphere with the effect of various fluid variables and with the various techniques. Satya Prasad et al. [32], Dodaa Ramya et al. [33,34], Reddy et al. [35], Srinivasa Raju and his co-authors [36-38] studied fluid flow problems on MHD flows on vertical plates and sheets. Jayalakshmamma et al. [39] explored the approximate study of the axisymmetric, steady flow of incompressible micropolar fluid and the impervious sphere is obtainable by presumptuous uniform flow faraway from the sphere. Dinesh et al. [40] studied the consistent flow past a spherical solid core inserted in another circular permeable medium.

Hence motivated by the above reference works, the existing examination of the traditional Newtonian liquid model has been updated to comprise the combined impact of Schmidt number and thermal radiative on Magnetohydrodynamics fluid flow of free convection around a Sphere. The varieties of stream fields for different relevant boundaries are talked about by utilizing a few diagrams. Examination of past writing results and close arrangement is noted. It is trusted that the accomplished outcomes dependent on the computational plan won't just give significant data to applications yet, in addition, fill in as an establishment for contemplating other related frameworks in designing and mechanical applications.

\section{Materials and Methods}

\subsection{Modelling of the problem.}

In this research work, the joint effects of Schmidt number and thermal radiative on MHD, steady-state free convective flow towards a sphere in the occurrence of Newtonian fluid, and homogeneous of first-order chemical reaction with the uniform magnetic field is studied. The actual model and framework of this research are presented in Fig. 1.

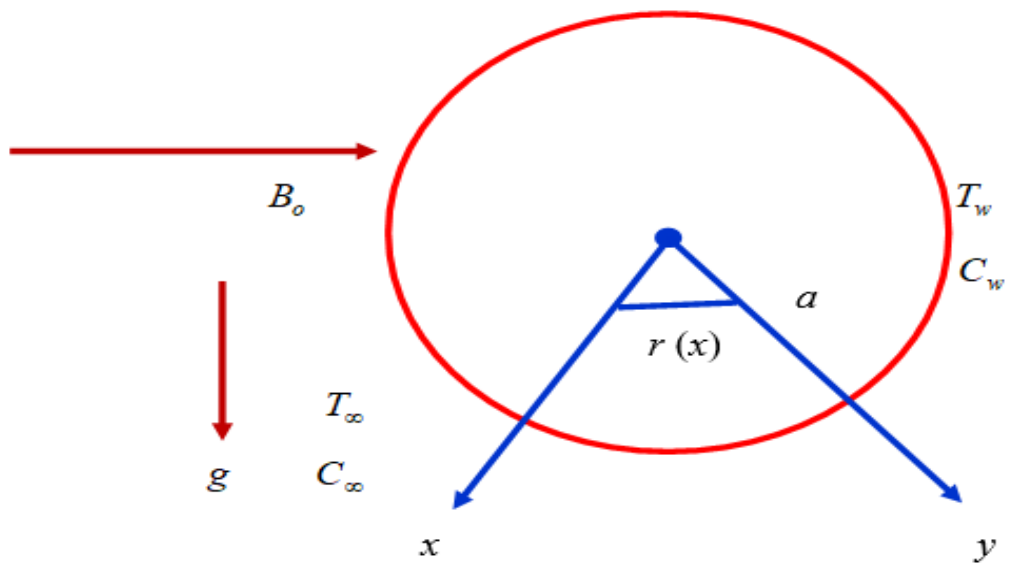

Figure 1. The actual model and facilitate framework.

For this investigation, the following assumptions are made. 
i. let $x, y$ and $r=r(x)$ are the Cartesian coordinates and the radial distance from the symmetrical axis to the sphere's surface, respectively.

ii. let $u, v, T, C$ are the fluid $x$-component velocity, $y$-component velocity, temperature, and solute concentration, respectively.

iii. The actual possessions and rate of chemical response $K c$ are steady entire the fluid.

iv. Induced magnetic field is thought to be tiny when contrasted with the applied magnetic field and is ignored.

v. The extending surface is kept up at endorsed surface temperature $T=T_{w}$ and solute concentration $C=C_{w}$ at $y=0$ in the recommended surface temperature and solute concentration limit condition and $T_{\infty}$ and $C_{\infty}$ are the temperature and concentration of the fluid far away from the surface.

Under the presumptions and according to Boussinesq's estimation, the governing models ([41] and [42]) are given by:

Continuity Equation:

$$
\frac{\partial}{\partial x}(r u)+\frac{\partial}{\partial y}(r v)=0
$$

Momentum Equation:

$u\left(\frac{\partial u}{\partial x}\right)+v\left(\frac{\partial u}{\partial y}\right)=v\left(\frac{\partial^{2} u}{\partial y^{2}}\right)+\left[g \beta_{T}\left(T-T_{\infty}\right)+g \beta_{C}\left(C-C_{\infty}\right)\right] \sin \left(\frac{x}{a}\right)-\left(\frac{\sigma B_{o}^{2}}{\rho}\right) u$

Equation of thermal energy:

$$
u\left(\frac{\partial T}{\partial x}\right)+v\left(\frac{\partial T}{\partial y}\right)=\frac{\kappa}{\rho C_{p}}\left(\frac{\partial^{2} T}{\partial y^{2}}\right)-\frac{1}{\rho C_{p}}\left(\frac{\partial q_{r}}{\partial y}\right)
$$

Equation of species concentration:

$$
u\left(\frac{\partial C}{\partial x}\right)+v\left(\frac{\partial C}{\partial y}\right)=D\left(\frac{\partial^{2} C}{\partial y^{2}}\right)-K c\left(C-C_{\infty}\right)
$$

The initial and boundary circumstances associated with this flow are

$$
\left.\begin{array}{l}
u=0, v=0, T=T_{w}, C=C_{w} \text { at } y=0 \\
u \rightarrow 0, T \rightarrow T_{\infty}, C \rightarrow C_{\infty} \text { as } y \rightarrow \infty
\end{array}\right\}
$$

The radial length from the regular axis to the exterior of the sphere $r=r(x)$ is defined by

$$
r=a \sin \left(\frac{x}{a}\right)
$$

The radiative heat transition term and rearranged with utilizing the Rosseland estimate (Sparrow and Cess [43]) is

$$
q_{r}=-\left(\frac{4 \sigma_{o}}{3 k^{*}}\right) \frac{\partial T^{4}}{\partial y}
$$

According to the Taylor series expansion, $T^{4}$ obtained as

$$
T^{4}=4 T_{\infty}^{3} T-3 T_{\infty}^{4}
$$

Eq.'s (7) and (8) are sub in equation (3), we obtain

$$
u\left(\frac{\partial T}{\partial x}\right)+v\left(\frac{\partial T}{\partial y}\right)=\frac{v}{\operatorname{Pr}}\left(1+\frac{4}{3 R}\right)
$$


By using similarity transformations

$$
\left.\begin{array}{l}
\theta(\eta)=\frac{T-T_{\infty}}{T_{w}-T_{\infty}}, \phi(\eta)=\frac{C-C_{\infty}}{C_{w}-C_{\infty}}, G r=\frac{g \beta_{T}\left(T_{w}-T_{\infty}\right) a^{3}}{v^{2}}, \\
\zeta=\frac{x}{a}, \eta=G r^{\frac{1}{4}} \frac{y}{a}, U=\frac{a}{v} G r^{\frac{-1}{2}} u, V=\frac{a}{v} G r^{\frac{-1}{4}} v
\end{array}\right\}
$$

The expressions (1), (2), (4) and (9) yields the dimensionless equations as:

$$
\begin{aligned}
& \frac{\partial}{\partial \zeta}(r U)+\frac{\partial}{\partial \eta}(r V)=0 \\
& U\left(\frac{\partial U}{\partial \zeta}\right)+V\left(\frac{\partial U}{\partial \eta}\right)=\frac{\partial^{2} U}{\partial \eta^{2}}+(\theta+N \phi) \sin \zeta-M U \\
& U\left(\frac{\partial \theta}{\partial \zeta}\right)+V\left(\frac{\partial \theta}{\partial \eta}\right)=\frac{1}{\operatorname{Pr}}\left(1+\frac{4}{3 R}\right) \frac{\partial^{2} \theta}{\partial \eta^{2}} \\
& U\left(\frac{\partial \phi}{\partial \zeta}\right)+V\left(\frac{\partial \phi}{\partial \eta}\right)=\frac{1}{S c}\left(\frac{\partial^{2} \phi}{\partial \eta^{2}}\right)-\gamma \phi
\end{aligned}
$$

Where $M=\frac{\sigma B_{o}^{2} a^{2}}{\rho v G r^{\frac{1}{2}}}$ and $\gamma=\frac{K c a^{2}}{\nu G r^{\frac{1}{2}}}$.

The dimensionless boundary conditions become:

$$
\left.\begin{array}{l}
U=0, V=0, \theta=1, \phi=1 \text { at } \eta=0 \\
U \rightarrow 0, \theta \rightarrow 0, \phi \rightarrow 0 \text { as } \eta \rightarrow \infty
\end{array}\right\}
$$

Assuming that $\psi(\zeta, \eta)=\zeta r(\zeta) f(\zeta, \eta)$

We obtained the following equations from above equations

$$
\begin{aligned}
& \frac{\partial^{3} f}{\partial \eta^{3}}+\left(1+\frac{\zeta}{\sin \zeta} \cos \zeta\right) f \frac{\partial^{2} f}{\partial \eta^{2}}-\left(\frac{\partial f}{\partial \eta}\right)^{2}-M\left(\frac{\partial f}{\partial \eta}\right)+\frac{\sin \zeta}{\zeta}(\theta+N \phi)=\zeta\left[\left(\frac{\partial f}{\partial \eta}\right) \frac{\partial^{2} f}{\partial \eta \partial \zeta}-\left(\frac{\partial f}{\partial \zeta}\right) \frac{\partial^{2} f}{\partial \eta^{2}}\right] \\
& \frac{1}{\operatorname{Pr}}\left(1+\frac{4}{3 R}\right)\left(\frac{\partial^{2} \theta}{\partial \eta^{2}}\right)+\left(1+\frac{\zeta}{\sin \zeta} \cos \zeta\right) f \frac{\partial \theta}{\partial \eta}=\zeta\left[\left(\frac{\partial f}{\partial \eta}\right) \frac{\partial \theta}{\partial \zeta}-\left(\frac{\partial f}{\partial \zeta}\right) \frac{\partial \theta}{\partial \eta}\right] \\
& \frac{1}{S c}\left(\frac{\partial^{2} \phi}{\partial \eta^{2}}\right)+\left(1+\frac{\zeta}{\sin \zeta} \cos \zeta\right) f \frac{\partial \phi}{\partial \eta}-\gamma \phi=\zeta\left[\left(\frac{\partial f}{\partial \eta}\right) \frac{\partial \phi}{\partial \zeta}-\left(\frac{\partial f}{\partial \zeta}\right) \frac{\partial \phi}{\partial \eta}\right]
\end{aligned}
$$

It tends to be observed that at the inferior stagnation purpose of the sphere $(\zeta \approx 0)$, the overhead models and circumstances reduce to the accompanying standard differential equations.

$$
\begin{aligned}
& f^{\prime \prime \prime}+2 f f^{\prime \prime}-f^{\prime 2}+(\theta+N \phi)-M f^{\prime}=0 \\
& \frac{1}{\operatorname{Pr}}\left(1+\frac{4}{3 R}\right) \theta^{\prime \prime}+2 f \theta^{\prime}=0 \\
& \frac{1}{S c} \phi^{\prime \prime}-\gamma \phi+2 f \phi^{\prime}=0
\end{aligned}
$$

and the boundary circumstances (6) become 


$$
\left.\begin{array}{l}
f=0, \quad f^{\prime}=0, \quad \theta=1, \quad \phi=1 \text { at } \eta=0 \\
f^{\prime} \rightarrow 0, \quad \theta \rightarrow 0, \quad \phi \rightarrow 0 \text { as } \eta \rightarrow \infty
\end{array}\right\}
$$

Of special significance for this type of flow and heat and mass transfer situation are the skin-friction coefficient $(C f)$, the Nusselt number $(N u)$ or heat transmission rate, and the Sherwood number $(S h)$ or the mass transmission rate. These physical amounts are characterized in dimensionless forms, given below.

$$
C f=\frac{G r^{\frac{-3}{4}} a^{2}}{\mu \nu} \tau_{w}=\zeta\left(\frac{\partial^{2} f}{\partial \eta^{2}}\right)_{\eta=0}
$$

where $\tau_{w}=\mu\left(\frac{\partial u}{\partial y}\right)_{y=0}$

$$
N u=\frac{G r^{\frac{-1}{4}} a}{\kappa\left(T_{w}-T_{\infty}\right)} q_{w}=-\left(1+\frac{4}{3 R}\right)\left[\theta^{\prime}(\eta)\right]_{\eta=0}
$$

where $q_{w}=-\kappa\left(\frac{\partial T}{\partial y}\right)_{y=0}$

$$
S h=\frac{G r^{\frac{-1}{4}} a}{\rho D\left(C_{w}-C_{\infty}\right)} m_{w}=-\left[\phi^{\prime}(\eta)\right]_{\eta=0}
$$

where $m_{w}=-\rho D\left(\frac{\partial C}{\partial y}\right)_{y=0}$

\section{Results and Discussion}

To tackle the arrangement of ordinary differential equations (20)-(22) with the initial and frontier conditions (23) mathematically, the space $[0, \infty)$ has been fulfilled by the entire region $\left[0, \eta_{\infty}\right]$ where $\eta_{\infty}$ is an appropriate real number that satisfies the entire domain. Additionally, (20)-(22) structure an exceptionally nonlinear coupled differential equations with boundary conditions. Consequently, (20)-(22) have been diminished to an arrangement of a few first-order initial value problems of seven dependent functions as

$$
\left.f=y_{1}, f^{\prime}=y_{2}, f^{\prime \prime}=y_{3}, \theta=y_{4}, \theta^{\prime}=y_{5}, \phi=y_{6}, \phi^{\prime}=y_{7}\right\}
$$

Subsequently, we build up the best mathematical procedure following the fourth-order Runge-Kutta Fehlberg algorithm through the shooting strategy. The emblematic programming MAPLE is utilized to acquire the most accurate solution. To tackle this framework, we require seven starting conditions though we have just four initial conditions for $f(0), f^{\prime}(0), \theta(0)$ and $\phi(0)$, while the other three $f^{\prime \prime}(0), \theta^{\prime}(0)$ and $\phi^{\prime}(0)$ were not given; henceforth,, we utilize computational shooting strategy where these three conditions are speculated to deliver the necessary three closure boundary conditions. During the numerical reproduction, the progression size is to be $\Delta \eta=0.001$ to get the desired solution and maintaining the accuracy $10^{-8}$. We taken, , $\eta_{\max }=10$ such that the numerical solution converges which satisfies all the conditions. The resulting algorithm is imagined in Fig. 2. 


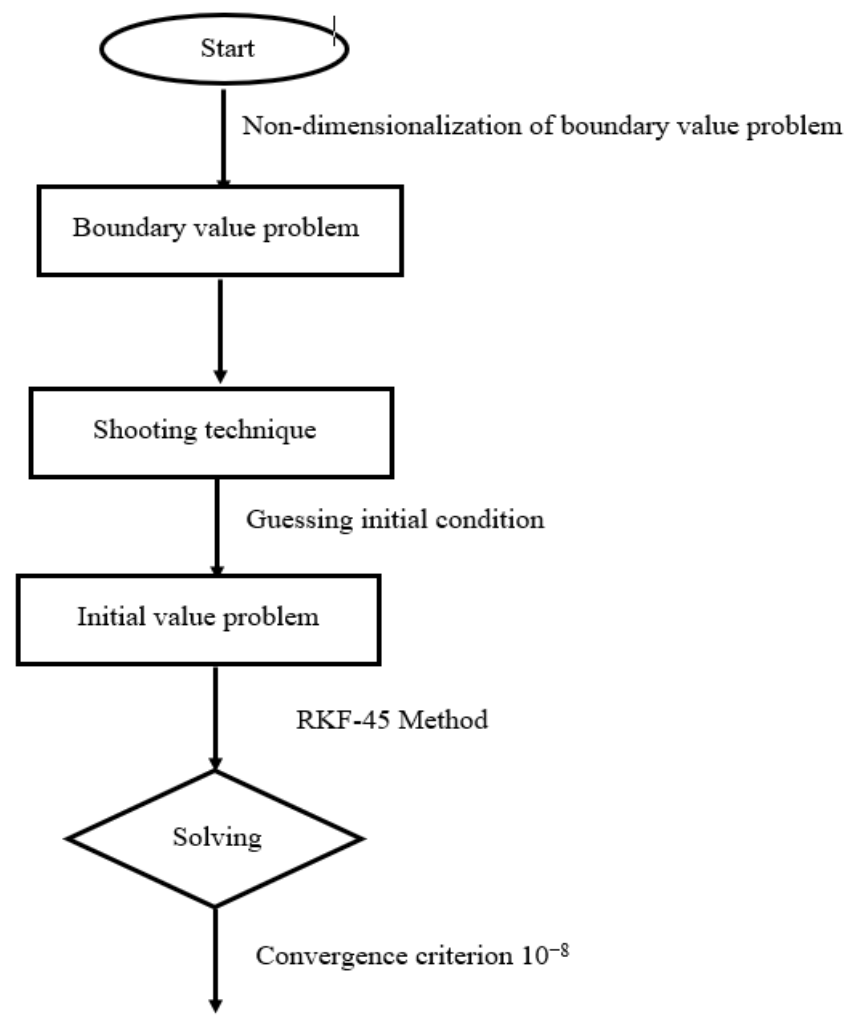

Figure 2. Flow diagram of the numerical procedure.

\subsection{Program code validation.}

The program code validation of this research work is discussed in table 1 . From this table, the present results $N u / G r^{1 / 4}$ are contrasted with the earlier existed results of Huang [41] and Cheng [42] at $\mathrm{M}=0, \mathrm{~N}=0$ and $\mathrm{R} \rightarrow \infty$. It tends to be seen from this table that amazing understanding was observed.

Table 1. Comparison of the numerical values of $N u / G r^{1 / 4}$ various values of $\zeta$.

\begin{tabular}{l|c|c|c}
$\zeta$ & Results of Huang [41] & Results of Cheng [42] & Present numerical results \\
\hline 0.0 & 0.4574 & 0.4576 & 0.448521345821 \\
\hline 0.1745 & 0.4563 & 0.4565 & 0.443002651862 \\
\hline 0.3491 & 0.4532 & 0.4534 & 0.443210042157 \\
\hline 0.5236 & 0.4480 & 0.4481 & 0.436255178441 \\
\hline 0.6981 & 0.4407 & 0.4407 & 0.439920061547 \\
\hline 0.8727 & 0.4312 & 0.4310 & 0.429330166524 \\
\hline 1.0470 & 0.4194 & 0.4191 & 0.409032100154 \\
\hline 0.2220 & 0.4053 & 0.4049 & 0398220155036 \\
\hline 0.3960 & 0.3886 & 0.3881 & 0.375326503048 \\
\hline 1.5710 & 0.3684 & 0.3686 & 0.359863146277
\end{tabular}

\subsection{Results and discussion.}

In this investigation, the influence of numerous pertinent constraints, namely, Magnetic field (M), Thermal radiation (R), Prandtl number $(\mathrm{Pr})$ and Chemical reaction $(\gamma)$ parameters, and Prandtl (Pr) and Schmidt (Sc) numbers on momentum, energy, and concentration profiles are illustrated through the figures Fig's. 3-11. 


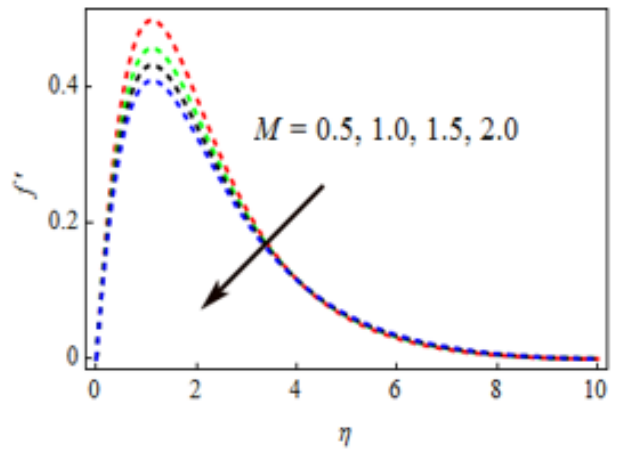

Figure 3. Impact of $M$ on velocity outlines.

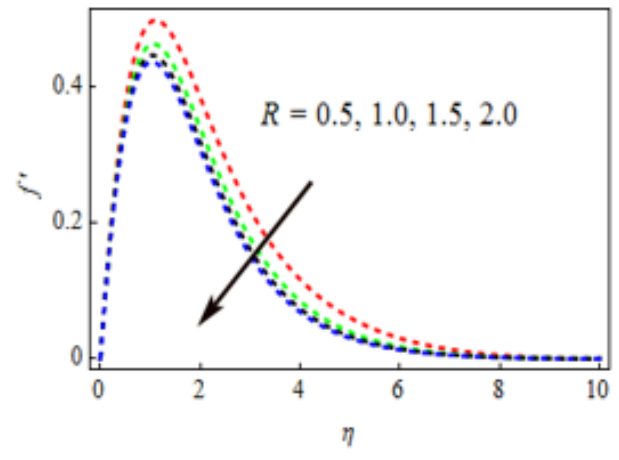

Figure 4. Impact of $R$ on velocity outlines.

Also, the influence of relevant constraints on drag force, rate of heat, and mass transmission are discussed through the related coefficients in Tables 2 and 3. Fig. 3 describes the inspiration of the Magnetic field parameter $(M)$ on fluid velocity profiles. MHD primary role utilized as a specialist is to control the boundary layer width. MHD chief utilized as a specialist to control the limit layer thickness. True to form, expanding the estimation of $M$, it diminishes the fluid velocity amazingly. Further, it tends to be seen that the thickness of the boundary layer and diminishing in the primary free stream district.

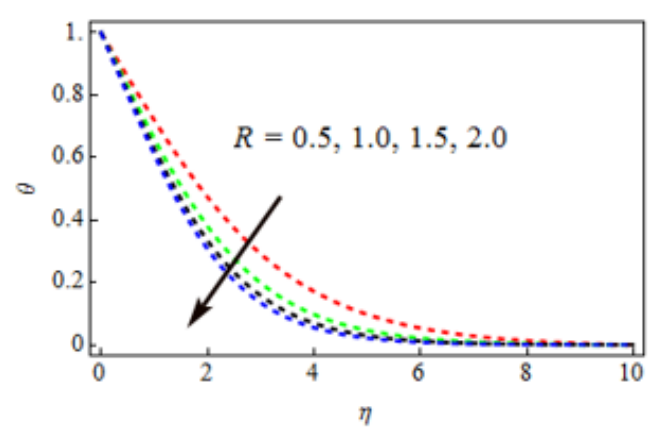

Figure 5. Impact of R on temperature outlines.

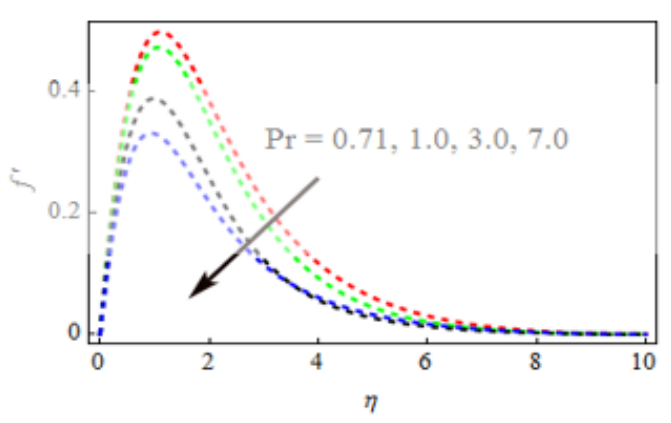

Figure 6. Impact of Pr on velocity outlines.

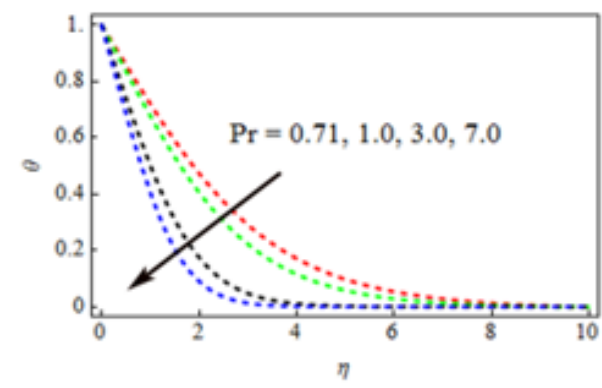

Figure 7. Impact of Pr on temperature outlines. https://biointerfaceresearch.com/

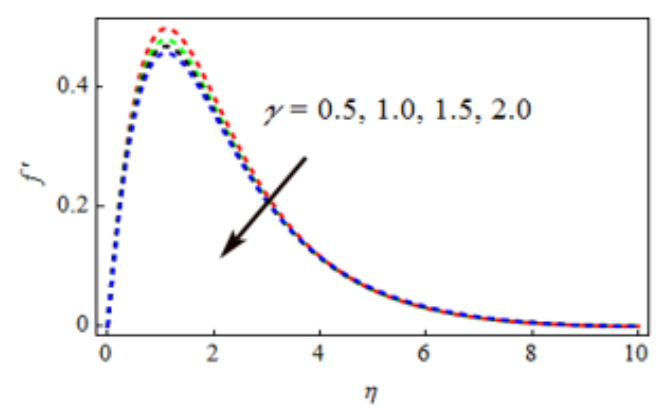

Figure 8. Impact of $\gamma$ on velocity outlines.

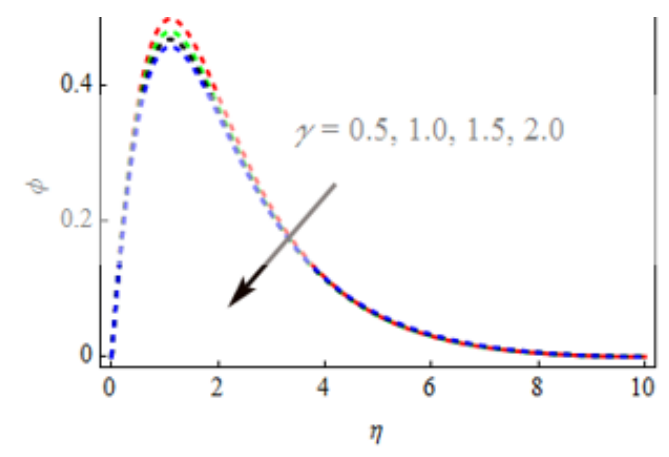

Figure 9. Impact of $\gamma$ on concentration outlines.

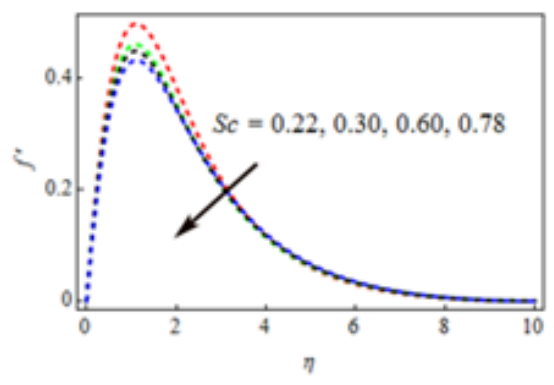

Figure 10. Impact of Sc on velocity outlines. 


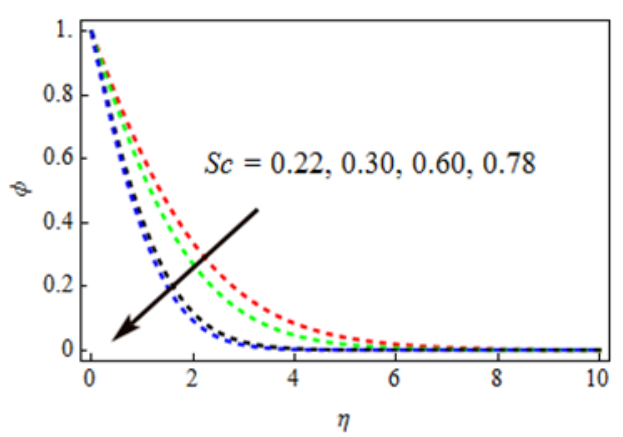

Figure 11. Impact of Sc on concentration outlines.

This happened because of the explanation that drags force acting inversely to the fluid flow and decreases fluid flow.

The impression of the thermal radiation boundary on the dimensionless velocity and dimensionless temperature appears in Figs. 4 and 5 separately. Fig. 4 demonstrates the velocity segment diminishes with an increment in the thermal radiation. Fig. 5 shows that the temperature diminishes with the enhancement of thermal radiation. The result is subjectively concurring with assumptions since the impact of radiation is to diminish the rate of the temperature of the fluid, consequently diminishing the temperature of the fluid. Fig. 6 explored the impact of Prandtl (Pr) number on fluid velocity outlines. It is seen that the expanding estimation of $\operatorname{Pr}$ brings about a decline in the velocity of the fluid. This is a direct result of the way that higher viscosity of liquid with the huge quantity of Prandtl number and a little thermal conductivity. This interferes with the velocity of the liquid and makes the fluid thicker. The influence of Prandtl ( $P r)$ number on fluid temperature outlines, as demonstrated in Fig. 7, is delineated as decelerating the temperature profile for mounting estimations of Pr. Thermal conductivity is low, and viscosity is high at a high Prandtl number. Growing the values of Pr reduces the thermal diffusivity, and the resulting hot liquid flows below, and the thermal frontier layer thickness diminishes with an enhancement of Prandtl number.

Fig. 8 and 9 explore the impact of the first-order chemical response parameter $(\gamma)$ on both the velocity and concentration outlines in the frontier layer. Expanding the chemical response parameter diminishes the fluid concentration. Continuously, the concentration of fluid decreases as an increment of $\gamma$. As a result, the lower the flow along with the plate, the decreasing the velocity of the fluid in the frontier layer. The impression of Schmidt number (Sc) on fluid velocity and concentration outlines are discussed in Figs. 10 and 11. From these figures, evident that increasing the $S c$ causes reductions in both the velocity and concentration outlines due to the decrease in the molecular flow. Furthermore, the thickness of the boundary layer of the concentration decreases as Sc increases. The influence of appropriate parameters viz., Magnetic field $(M)$, Thermal radiation $(R)$, and Chemical reaction $(\gamma)$ parameters, Prandtl $(P r)$, and Schmidt $(S c)$ number on Skin-friction coefficient is shown in table 2; the Skin-friction coefficient is decreasing with growing values of Magnetic field $(M)$, Thermal radiation $(R)$, Chemical reaction $(\gamma)$ parameters and Prandtl $(P r)$, and Schmidt $(S c)$ numbers.

Table 2. Numerical values of the Skin-friction coefficient for the effects of M, Pr, R, Sc, and $\gamma$.

\begin{tabular}{l|c|c|c|c|c}
$\boldsymbol{M}$ & $\mathbf{P r}$ & $\boldsymbol{R}$ & $\boldsymbol{S} \boldsymbol{c}$ & $\boldsymbol{\gamma}$ & $\boldsymbol{C} \boldsymbol{f}$ \\
\hline 0.5 & 0.71 & 0.5 & 0.22 & 0.5 & 0.425332615827 \\
\hline $\mathbf{1 . 0}$ & 0.71 & 0.5 & 0.22 & 0.5 & 0.376002315846 \\
\hline 0.5 & $\mathbf{7 . 0 0}$ & 0.5 & 0.22 & 0.5 & 0.346310045209 \\
\hline 0.5 & 0.71 & $\mathbf{1 . 0}$ & 0.22 & 0.5 & 0.386452275013 \\
\hline 0.5 & 0.71 & 0.5 & $\mathbf{0 . 3 0}$ & 0.5 & 0.354203169987 \\
\hline 0.5 & 0.71 & 0.5 & 0.22 & $\mathbf{1 . 0}$ & 0.396230124823
\end{tabular}


The effects of emerging parameters, namely Prandtl number $(\operatorname{Pr})$ and Thermal radiation (R) on Nusselt number coefficient and the effects of Schmidt number (Sc), Chemical reaction parameter $(\gamma)$ on Sherwood number coefficient are demonstrated in table 3. Because of table 3, the Nusselt number coefficient decreases with enhancing Prandtl number (Pr) and Thermal radiation $(\mathrm{R})$ values. From this table, it is also observed that the Sherwood number coefficient is decreasing with enhancing values of Schmidt $(S c)$ number and Chemical reaction $(\gamma)$ parameter.

Table 3. Numerical values of Nusselt and Sherwood number coefficients.

\begin{tabular}{l|c|c|c|c|c}
$\mathbf{P r}$ & $\boldsymbol{R}$ & $\boldsymbol{N u}$ & $\boldsymbol{S c}$ & $\boldsymbol{\gamma}$ & $\boldsymbol{S h}$ \\
\hline 0.71 & 0.5 & 0.125336152848 & 0.22 & 0.5 & 0.153998002785 \\
\hline $\mathbf{7 . 0 0}$ & 0.5 & 0.082031544786 & $\mathbf{0 . 3 0}$ & 0.5 & 0.093326155402 \\
\hline 0.71 & $\mathbf{1 . 0}$ & 0.103264722487 & 0.22 & $\mathbf{1 . 0}$ & $0.112003487562-$
\end{tabular}

\section{Conclusions}

In this research work, the combined influence of the Chemical reaction parameter and Schmidt number on steady and MHD fluid flow on the sphere with thermal radiation is considered. The basic leading equations are taken for this fluid flow. The computational solutions of differential equations are obtained by applying the fourth-order Runge-KuttaFehlberg strategy through the shooting method using MATHEMATICA programming. The important results of the above study are presented below: The velocity profiles fall with an enhancing of Magnetic field and Thermal radiation parameters; Increasing the values of the Prandtl number heightens the velocity and temperature of the fluid in the boundary layer regime; The velocity and Concentration profiles decrease with the increasing values of the Chemical reaction parameter; An enhancement in the Schmidt number leads to enhance the thermal boundary layer thickness; The species concentration of fluid reduces with enhancing the values of Schmidt number; More so, the results are compared by already distributed works and are in great agreement.

\section{Funding}

The authors claim that they had no financing support and no funding.

\section{Acknowledgments}

This research has no acknowledgment.

\section{Conflicts of Interest}

The authors claim that they are unaware of the competitive financial benefits or personal relationships that appear to affect the work reported in this paper.

\section{References}

1. Ahmadi, M.; Willing, G. Heat transfer measurement in water based nanofluids. International Journal of Heat and Mass Transfer 2018, 118, 40-47, https://doi.org/10.1016/j.ijheatmasstransfer.2017.10.090.

2. Naseem, A.; Shafiq, A.; Zhao, L.; Farooq, M.U. Analytical investigation of third grade nanofluidic flow over a Riga plate using Cattaneo-Christov model. Results in Physics 2018, 9, 961-969, https://doi.org/10.1016/j.rinp.2018.01.013.

3. Ismail, N.S.; Arifin, N.M.; Nazar, R.; Bachok, N. Stability analysis of unsteady MHD stagnation point flow and heat transfer over a shrinking sheet in the presence of viscous dissipation. Chinese Journal of Physics 2019, 57, 116-126, https://doi.org/10.1016/j.cjph.2018.12.005. 
4. Soomro, F.A.; Haq, R.U.; Al-Mdallal, Q.M.; Zhang, Q. Heat generation/absorption and nonlinear radiation effects on stagnation point flow of nanofluid along a moving surface. Results Physics 2018, 8, 404-414, https://doi.org/10.1016/j.rinp.2017.12.037.

5. Ghalambaz, M.; Sheremet, M.A.; Mehryan, S.A.M.; Kashkooli, F.M.; Pop, I. Local thermal non-equilibrium analysis of conjugate free convection within a porous enclosure occupied with $\mathrm{Ag}-\mathrm{MgO}$ hybrid nanofluid. Journal of Thermal Analysis and Calorimetry 2019, 135, 1381-1398, https://doi.org/10.1007/s10973-0187472-8.

6. Veera Krishna, M.; Chamkha, A.J. Hall and ion slip effects on MHD rotating boundary layer flow of nanofluid past an infinite vertical plate embedded in a porous medium. Results in Physics 2019, 15, https://doi.org/10.1016/j.rinp.2019.102652.

7. Thameem Basha, H.; Sivaraj, R.; Subramanyam Reddy, A.; Chamkha, A.J. SWCNH/diamond-ethylene glycol nanofluid flow over a wedge, plate and stagnation point with induced magnetic field and nonlinear radiation-solar energy application. The European Physical Journal Special Topics 2019, 228, 2531-2551, https://doi.org/10.1140/epjst/e2019-900048-x.

8. Kumar, B.; Seth, G.S.; Nandkeolyar, R.; Chamkha, A.J. Outlining the impact of induced magnetic field and thermal radiation on magneto-convection flow of dissipative fluid. International Journal of Thermal Sciences 2019, 146, https://doi.org/10.1016/j.ijthermalsci.2019.106101.

9. Veera Krishna, M.; Chamkha, A.J. Hall and ion slip effects on MHD rotating flow of elastico-viscous fluid through porous medium. International Communications in Heat and Mass Transfer 2020, 113, https://doi.org/10.1016/j.icheatmasstransfer.2020.104494.

10. Veera Krishna, M.; Ahamad, N.A.; Chamkha, A.J. Hall and ion slip effects on unsteady MHD free convective rotating flow through a saturated porous medium over an exponential accelerated plate. Alexandria Engineering Journal 2020, 59, 565-577, https://doi.org/10.1016/j.aej.2020.01.043.

11. Ramesh, G.K.; Shehzad, S.A.; Rauf, A.; Chamkha, A.J. Heat transport analysis of aluminum alloy and magnetite graphene oxide through permeable cylinder with heat source/sink. Physica Scripta 2020, 95, https://doi.org/10.1088/1402-4896/aba5af.

12. Menni, Y.; Chamkha, A.J.; Massarotti, N.; Ameur, H.; Kaid, N.; Bensafi, M. Hydrodynamic and thermal analysis of water, ethylene glycol and water-ethylene glycol as base fluids dispersed by aluminum oxide nano-sized solid particles. International Journal of Numerical Methods for Heat \& Fluid Flow 2020, 30, 4349-4386, https://doi.org/10.1108/HFF-10-2019-0739.

13. Ganesh Kumar, K.; Gnaneswara Reddy, M.; Sudharani, M.V.V.N.L.: Shehzad, S.A.; Chamkha, A.J. Cattaneo-Christov heat diffusion phenomenon in Reiner-Philippoff fluid through a transverse magnetic field. Physica A: Statistical Mechanics and its Applications, 2020, 541, https://doi.org/10.1016/j.physa.2019.123330.

14. Wakif, A.; Chamkha, A.J.; Animasaun, I.L.; Zaydan, M.; Waqas, H.; Sehaqui, R. Novel physical insights into the thermodynamic irreversibilities with in dissipative MHD fluid flows past over a moving horizontal riga plate in the coexistence of wall suction and joule heating effects: A comprehensive numerical investigation. Arabian Journal of Engineering and Science 2020, 45, 9423-9438, https://doi.org/10.1007/s13369-020-04757-3.

15. Bhowmik, H.; Gharibia, A.; Yaarubia, A.; Alawia, N. Transient natural convection heat transfer analyses from a horizontal cylinder. Case Studies in Thermal Engineering 2019, 14, https://doi.org/10.1016/j.csite.2019.100422.

16. Jena, S.; Dash, G.C.; Mishra, S.R. Chemical reaction effect on MHD viscoelastic fluid flow over a vertical stretching sheet with heat source/sink. Ain Shams Engineering Journal 2018, 9, 1205-1213, https://doi.org/10.1016/j.asej.2016.06.014.

17. Anil Kumar, M.; Dharmendar Reddy, Y.; Shankar Goud, B.; Srinivasa Rao, V. Effects of soret, dufour, hall current and rotation on MHD natural convective heat and mass transfer flow past an accelerated vertical plate through a porous medium. International Journal of thermo fluids 2021, 9, https://doi.org/10.1016/j.ijft.2020.100061.

18. Shankar Goud, B.; Dharmendar Reddy, Y.; Srinivasa Rao, V. Thermal radiation and Joule heating effects on a magnetohydrodynamic Casson nanofluid flow in the presence of chemical reaction through a nonlinear inclined porous stretching sheet. Journal of Architecture and Marine Engineering 2020, 17, 143-164, https://dx.doi.org/10.3329/jname.v17i2.49978.

19. Veera Krishna, M.; Swarnalathamma, B.V.; Prakash, J. Heat and mass transfer on unsteady MHD Oscillatory flow of blood through porous arteriole. Applied Fluid Dynamics: Lecture Notes in Mechanical Engineering 2018, 22, 207-224, https://doi.org/10.1007/978-981-10-5329-0_14.

20. Seth, G.S.; Bhattacharyya, A.; Kumar, R.; Mishra, M.K. Modelling and numerical simulation of hydromagnetic natural convection Casson fluid flow with nth order chemical reaction and Newtonian heating in porous medium. Journal of Porous Media 2019, 22, 1141-1157, https://doi.org/10.1615/JPorMedia.2019025699.

21. Sahaya Jenifer, A.; Saikrishnan, P.; Lewis, R.W. Unsteady MHD Mixed Convection Flow of Water over a Sphere with Mass Transfer. Journal of Applied and Computational Mechanics 2021, 7, 935-943, https://doi.org/10.22055/JACM.2021.35920.2761. 
22. Widodo, B.; Abu, M.; Imron, C. Unsteady nano fluid flow through magnetic porous sphere under the influence of mixed convection. Journal of Physics: Conference Series 2019, 1153, https://doi.org/10.1088/1742-6596/1153/1/012053.

23. Widodo, B. Magnetohydrodynamics fluid flow passing through a sliced magnetic sphere influenced by mixed convection. Journal of Physics: Conference Series 2021, 1836, https://doi.org/10.1088/17426596/1836/1/012042.

24. Madasu, K.P.; Bucha, T. Influence of MHD on micropolar fluid flow past a sphere implanted in porous media. Indian Journal of Physics 2021, 95, 1175-1183, https://doi.org/10.1007/s12648-020-01759-7.

25. Veera Krishna, M.; Ameer Ahamad, N.; Chamkha, A.J. Radiation absorption on MHD convective flow of nanofluids through vertically travelling absorbent plate. Ain Shams Engineering Journal 2021, 12, 30433056, https://doi.org/10.1016/j.asej.2020.10.028.

26. Patil, P.M.; Shankar, H.F.; Hiremath, P.S.; Momoniat, E. Nonlinear mixed convective nanofluid flow about a rough sphere with the diffusion of liquid hydrogen. Alexandria Engineering Journal 2021, 60, 1043-1053, https://doi.org/10.1016/j.aej.2020.10.029.

27. Swalmeh, M.Z. Numerical solutions of hybrid nanofluids flow via free convection over a solid sphere. Journal of Advanced Research in Fluid Mechanics and Thermal Sciences 2021, 83, 34-45, https://doi.org/10.37934/arfmts.83.1.3445.

28. Figueroa, A.; Rivero, M.; Núñez, J.; Rojas, J.; Rivera, I. Oscillatory flow between concentric spheres driven by an electromagnetic force. Journal of Fluid Mechanics 2021, 920, https://doi.org/10.1017/jfm.2021.397.

29. Gul, T.; Ali, B.; Alghamdi, W.; Nasir, S.; Saeed, A.; Kumam, P.; Mukhtar, S.; Kumam, W.; Jawad, M. Mixed convection stagnation point flow of the blood based hybrid nanofluid around a rotating sphere. Scientific Reports 2021, 11, https://doi.org/10.1038/s41598-021-86868-X.

30. Garnayak, S.; Rath, S. Numerical Computation on Natural Convection Heat Transfer From an Isothermal Sphere With Semicircular Ribs. Journal of Heat Transfer 2021, 143, https://doi.org/10.1115/1.4051603.

31. Mahdy, A.; Siddiqa, S.; Nabwey, H.A. Impact Of Gyrotactic Microorganisms On Natural Nanofluid Bioconvection Flow By A Sphere Immersed In Porous Media. 2021, 24, 65-81, https://doi.org/10.1615/JPorMedia.2021038054.

32. Satya Prasad, M.; Swamy Naidu, N.V.; Srinivasa Raju, R. Characteristics of Magnetohydrodynamics Nanofluid Flow in the Presence of Porous Medium Towards a Stretching Sheet with a Convective Boundary Condition. Journal of Nanofluids 2020, 9, 346-353, https://doi.org/10.1166/jon.2020.1757.

33. Jithender Reddy, G.; Srinivasa Raju, R.; Manideep, P.; Anand Rao, J. Thermal diffusion and diffusion thermo effects on unsteady MHD fluid flow past a moving vertical plate embedded in porous medium in the presence of Hall current and rotating system. Transactions of A. Razmadze Mathematical Institute 2016, 170, 243-265, https://doi.org/10.1016/j.trmi.2016.07.001.

34. Ramya, D.; Raju, R.S.; Rao, J.A. Influence of Chemical Reaction on MHD Boundary Layer Flow of Nanofluids Over a Nonlinear Stretching Sheet with Thermal Radiation. Journal of Nanofluids 2016, 5, 880888, https://doi.org/10.1166/jon.2016.1276.

35. Ramya, D.; Raju, R.; Rao, J. Boundary Layer Viscous Flow of Nanofluids and Heat Transfer over a Nonlinearly Isothermal Stretching Sheet in the Presence of Heat Generation/Absorption and Slip Boundary Conditions. International Journal of Nanoscience and Nanotechnology 2016, 12, 251-268.

36. Raju, R.S.; Jithender Reddy, G.; Rao, J.A.; Rashidi, M.M. Thermal diffusion and diffusion thermo effects on an unsteady heat and mass transfer magnetohydrodynamic natural convection Couette flow using FEM. Journal of Computational Design and Engineering 2016, 3, 349-362, https://doi.org/10.1016/j.jcde.2016.06.003.

37. Raju, R.; Aruna, G.; Varma, S. Chemically Reacting Fluid Flow over an Exponentially Accelerated Vertical Plate in a Magnetic Field and Variable Temperature via LTT and FEM. Theoretical and Applied Mechanics 2016, 43, 49-83, https://doi.org/10.2298/TAM151214003S.

38. Srinivasa Raju, R. Combined Influence of Thermal Diffusion and Diffusion Thermo on Unsteady MHD Free Convective Fluid Flow Past an Infinite Vertical Porous Plate in Presence of Chemical Reaction. Journal of The Institution of Engineers (India): Series C 2016, 97, 505-515, https://doi.org/10.1007/s40032-016-02585.

39. Jayalakshmamma, D.V.; Dinesh, P.A.; Chandrashekhar, D.V. Numerical Study of Micropolar Fluid Flow Past an Impervious Sphere. Defect and Diffusion Forum 2018, 388, 344-349, https://doi.org/10.4028/www.scientific.net/DDF.388.344.

40. Dinesh, P.A.; Jayalakshmamma, D.V.; Chandrashekhar, D.V. Exact solution for viscous fluid flow in porous medium with magnetic field, Sixth International Conference on Porous Media and Its Applications in Science, Engineering and Industry, Eds, ECI Symposium Series, 2016.

41. Huang, M.J.; Cha'o-Kuang, C. Laminar free convection from a sphere with blowing and suction. Journal of Heat transfer 1987, 109, 529-532, https://doi.org/10.1115/1.3248117.

42. Cheng, C.-Y. Natural convection heat and mass transfer from a sphere in micropolar fluids with constant wall temperature and concentration. International Communications in Heat and Mass Transfer 2008, 35, 750-755, https://doi.org/10.1016/j.icheatmasstransfer.2008.02.004.

43. Sparrow, E.; Cess, R. Radiation Heat Transfer, Hemisphere. Wahington, 1978. 